\title{
Rhabdomyolysis: Prolonged and high-intensity exercises, impact on renal function
}

\author{
Raphael Fabricio de Souza ${ }^{1,2 *}$ and Regina Souza Aires ${ }^{2}$ \\ ${ }^{1}$ Departamento de Educação Física, Universidade Federal de Sergipe - UFS, São Cristovão, Sergipe, Brazil \\ ${ }^{2}$ Departamento de Fisiologia e Farmacologia, Centro de Biociências, Universidade Federal de Pernambuco, Recife, Pernambuco, Brazil
}

\begin{abstract}
Rhabdomyolysis is a syndrome caused by injury to skeletal muscle that usually leads to acute kidney injury (AKI) and has been linked to different conditions, including severe trauma and intense physical exercise. The Cases related to vigorous physical exercise are observed in individuals in whom the severity it is due the response to the prolonged and high-intensity exercises. Additionally, the excessive use of Nonsteroidal anti-inflammatory drugs (NSAIDs), commonly used by endurance competitors can cause renal insufficiency. This short communication presents general aspects about rhabdomyolysis, pathophysiological mechanism, clinical manifestation and treatment.
\end{abstract}

\section{Introduction}

Rhabdomyolysis is a syndrome caused by the destruction of musculoskeletal cells, which releases intracellular substances toxic to the circulation, including myoglobin, which are harmful to the body. Typically, rhabdomyolysis presents with myalgia, muscle weakness and dark urine. Clinical manifestations may range from an asymptomatic disease to a life-threatening condition with very high enzymes, acute kidney injury (AKI) and electrolyte disturbances.

The syndrome is reported in athletes having a higher prevalence in men. Can be induced by prolonged and high-intensity exercises [1] or by sudden and excessive contractions of the skeletal muscles, with typical appearance of prominent clinical manifestations between 24 and 48 hours after the activity. Being more often reported in certain running modalities than in cycling and triathlon. This case, rhabdomyolysis is related to excessive muscle activity, changes in body temperature, electrolyte, endocrine and circulatory changes.

Dehydration may be a key factor in triggering the syndrome, even in previously healthy and well-conditioned practitioners. Intake of isotonic fluids should be prioritized since hypotonic fluids can lead to varying degrees of hyponatremia. Reduced sodium rates, by mechanisms not yet well elucidated, are pre-disposed to rhabdomyolysis.

\section{Clinical manifestations and treatment}

The clinical presentation is extremely variable. In conscious patients, the main complaint may be of sensitivity, pain, stiffness and cramps accompanied by weakness and loss of function. However, myalgia may be absent or minimal initially. In comatose patients, the observation of stiffness in the extremities may suggest rhabdomyolysis. Skin changes due to ischemic tissue damage (discoloration, blistering) may be present in the affected area. Physical examination may reveal edema in muscles that may worsen after parenteral rehydration. Severe muscle edema may result in compartment syndrome, with no pulse.

Dark urine (reddish chestnut) is the classic manifestation of rhabdomyolysis. Signs of dehydration caused by the sequestering of fluid by the affected muscles may be present along with oliguria. Signs related to the complications of rhabdomyolysis (hyperkalemia, acute renal failure, metabolic acidosis, disseminated intravascular coagulation and, rarely, respiratory failure) may also be present between clinical manifestations.

The treatment for rhabdomyolysis, if done immediately, usually does not cause sequelae and consists of the application of intravenous saline, absurd intake of fluids and administration of diuretics to dilute myoglobin in the blood and stimulate the kidneys with the production and release of urine. Depending on the patient's condition, more drastic procedures, such as hemodialysis or surgery to "unclog" may be applied [2].

\section{Molecular mechanisms in rhabdomyolysis}

The most accepted pathophysiological mechanism is that the deficit or even depletion of adenosine triphosphate due to strenuous exercise induces a dysfunction of Na-K-ATPase, impairing the redistribution of ionic balance that occurs physiologically during exercise. The increase in sodium concentration in myocytes causes the $\mathrm{Na}-\mathrm{Ca}$ exchanger to work to reverse this state, in order to accumulate calcium in the high ion concentrations in muscle fibers are associated with cytotoxic events such as: activation of proteases and phospholipases, higher mitochondrial, sarcoplasmic calcium rates and stimulation of apoptosis signaling cellular [3].

Common final pathophysiological mechanisms among these causes of rhabdomyolysis include an uncontrolled rise in free intracellular calcium and activation of calcium-dependent proteases, which lead

*Correspondence to: Raphael Fabricio de Souza. Departamento de Educação Física, Universidade Federal de Sergipe - UFS, São Cristovão, Sergipe, Brazil, Tel: 557931946600; E-mail: desouza@ufs.br; raphaelctba20@hotmail.com

Key words: exercise, renal failure, nephrology

Received: November 24, 2018; Accepted: December 05, 2018; Published: December 10, 2018 
to destruction of myofibrils and lysosomal digestion of muscle fiber contents [4]. Calcium is among the major ions involved in maintaining skeletal muscle homeostasis. On the other hand hyperkalemia may be important to the point of leading to cardiac arrhythmias, hyperphosphatemia, metabolic acidosis and oscillations in serum calcium, and depending on the magnitude of these complications, hemodialysis should be indicated.

Another fact, myoglobin is a low molecular weight protein that is freely filtered by the kidney, and when there is muscle damage its concentration in the bloodstream is high, when it is eliminated in high concentrations it gave a dark color to the urine. Normally, after the end of muscle injury myoglobin blood levels return to normal within a few hours due to hepatic metabolism and renal excretion. The problem is that myoglobin is toxic to the kidney, and although it does not cause kidney damage alone, together with decreased circulating blood volume and decreased kidney perfusion can lead to myoglobinuric AKI.

Finally, serum creatine kinase $(\mathrm{CK})$ is the most reliable diagnostic marker of rhabdomyolysis. Studies confirm the elevation CK that occurs during strenuous exercise, healthy subjects who participated in long marches (50 and $80 \mathrm{~km}$ ) with heavy load also showed a large increase of CK during each march, reaching levels 40 to 80 times higher than the typical value for healthy sedentary participants, reflecting continuous muscular damages [5].

\section{Renal failure}

Rhabdomyolysis produces multiple adverse effects on the kidney, such as vasoconstriction, oxidative stress, inflammation and apoptosis [6]. Renal vasoconstriction is caused by reduced renal blood flow due to excessive leakage of extracellular fluid into the damaged muscle cells and by secondary activation of the renin-angiotensin aldosterone system [7]. On the other hand, the key role of oxidative stress in acute kidney injury in rhabdomyolysis has also been demonstrated using antioxidants which effectively prevented oxidative renal damage and dysfunction [8].

The accumulation of myoglobin in the kidney is central in the mechanism that leads to kidney damage [9]. Myoglobin deposition associates with impairing the primary urine flow and reabsorption, apparently through induction of oxidative stress with subsequent nephron cell death [10]. Recent data show that myoglobin induces lipid peroxidation of cellular membranes ultimately producing malondialdehyde as well as isoprostanes [11].

In addition, it is agreed that renal failure is due to the combined effects of direct cytotoxicity due to the accumulation of renal tubular myoglobin, hypovolemia, and aciduria. Increased levels of uric acid may play an important role in the renal alterations induced by heat stress and continuous episodes of rhabdomyolysis. Therefore, treatments aimed to reduce hyperuricemia may help to decrease the renal burden in these conditions [12].

\section{Nonsteroidal anti-inflammatory drugs and AKI}

Nonsteroidal anti-inflammatory drugs (NSAIDs) permitted by the World Anti-Doping Agency, are commonly used by competitors including runners and ultra-marathon competitors to improve performance in endurance events performed over large distances. Recently a study [13] showed that high creatinine excretion correlated with NSAIDs (i.e. ibuprofen) intake indicating an increased risk of AKI in ultramarathoners. The excessive use of NSAIDs, can cause renal insufficiency, what will be aggravated during a test of long distance being able to increase the severity of the rhabdomyolysis.
The use of NSAIDs requires special attention because they inhibit the activity of satellite cells, potentially inhibiting muscle adaptation to exercise and cause renal hemodynamic changes including the stimulation of the renin-angiotensin aldosterone system, vasoconstriction, decreased renal blood flow, and water retention, which may lead to hyponatremia (i.e., low plasma sodium concentration) and increased stress on the kidneys during endurance competition [14].

\section{Conclusion}

This brief communication study illustrates the concern of exercise in prolonged and high intensity induction of AKI and rhabdomyolysis.

\section{Ethics approval}

Not applicable

\section{Consent for publication}

Not applicable

\section{Availability of data and materials}

Not applicable

\section{Competing interests}

The author declares that there are no competing interests

\section{Funding}

None

\section{References}

1. Lozowska D, Liewluck T, Quan D, Ringel SP (2015) Exertional rhabdomyolysis associated with high intensity exercise. Muscle Nerve 52: 1134-1135.

2. Venkataraman R, Kellum JA (2007) Prevention of acute renal failure. Chest 131: 300308. [Crossref]

3. Panizo N, Rubio-Navarro A, Amaro-Villalobos JM, Egido J, Moreno JA (2015) Molecular mechanisms and novel therapeutic approaches to rhabdomyolysis-induced acute kidney injury. Kidney Blood Press Res 40: 520-532.

4. Zhang MH (2012) Rhabdomyolosis and its pathogenesis. World J Emerg Med 3: 11 15. [Crossref]

5. Chevion S, Moran DS, Heled Y, Shani Y, Regev G, et al. (2003) Plasma antioxidant status and cell injury after severe physical exercise. Proc Natl Acad Sci 100: 51195123. [Crossref]

6. Scalco RS, Snoeck M, Quinlivan R, Treves S, Laforét P, et al. (2016) Exertional rhabdomyolysis: physiological response or manifestation of an underlying myopathy? BMJ Open Sport Exerc Med 2: e000151. [Crossref]

7. Petejova N, Martinek A (2014) Acute kidney injury due to rhabdomyolysis and renal replacement therapy: a critical review. Crit Care 18: 224.

8. Gois PHF, Canale D, Volpini RA, Ferreira D, Veras MM, et al. (2016) Allopurinol attenuates rhabdomyolysis-associated acute kidney injury: Renal and muscular protection. Free Radic Biol Med 101: 176-189. [Crossref]

9. Zorova LD, Pevzner IB, Chupyrkina AA, Zorov SD, Silachev DN, et al. (2016) The role of myoglobin degradation in nephrotoxicity after rhabdomyolysis. Chem Biol Interact 256: 64-70. [Crossref]

10. Boutaud O, Roberts LJ (2011) Mechanism-based therapeutic approaches to rhabdomyolysis-induced renal failure. Free Radic Biol Med 51: 1062-1067.

11. Reeder BJ, Sharpe MA, Kay AD, Kerr M, Moore K, et al. (2002) Toxicity of myoglobin and haemoglobin: oxidative stress in patients with rhabdomyolysis and subarachnoid haemorrhage. Biochem Soc Trans 30: 745-748.

12. Sánchez-Lozada LG, García-Arroyo FE, Gonzaga G, Silverio O, Blas-Marron MG, et al. (2018) Kidney injury from recurrent heat stress and rhabdomyolysis: Protective role of allopurinol and sodium bicarbonate. Am J Nephrol 48: 339-348. 
13. Lipman GS, Shea K, Christensen M, Phillips C, Burns P, et al. (2017) Ibuprofen versus placebo effect on acute kidney injury in ultramarathons: A randomised controlled trial. Emerg Med J 34: 637-642.
14. Fabricio de Souza R, Gama de Matos D, Pires Ferreira AR, Chilibeck P, de Almeida Barros N, et al. (2018) The effect of ibuprofen on muscle, hematological and renal function, hydric balance, pain, and performance during intense long-distance running. J strength Cond Res 29461420.

Copyright: (C2018 Fabricio de Souza R. This is an open-access article distributed under the terms of the Creative Commons Attribution License, which permits unrestricted use, distribution, and reproduction in any medium, provided the original author and source are credited. 